Supporting Information

\title{
Will any crap we put into graphene increase its electrocatalytic effect?
}

Lu Wang ${ }^{1}$, Zdenek Sofer ${ }^{2}$, Martin Pumera ${ }^{2,3,4 *}$

${ }^{1}$ Materials Chemistry and Nanochemistry Research Group, Solar Fuels Cluster, Departments of Chemistry, University of Toronto, 80 St. George Street, Toronto, ON M5S 3H6, Canada

${ }^{2}$ Center for Advanced Functional Nanorobots, Dept. of Inorganic Chemistry, University of Chemistry and Technology, Technicka 5, Praha 6166 28, Czech Republic

3 Future Energy and Innovation Laboratory, Central European Institute of Technology, Brno University of Technology, Purkyňova 656/123, Brno, CZ-616 00, Czech Republic.

${ }^{4}$ Department of Medical Research, China Medical University Hospital, China Medical University, No. 91 Hsueh-Shih Road, Taichung, Taiwan 


\section{Experimental Section}

Materials. $N, N$-dimethylformamide (DMF) was obtained from Sigma-Aldrich. Chicken guano was collected locally in the Czech Republic. Nitrogen of $99.9999 \%$ purity was obtained from SIAD.

Synthesis. Graphene oxide was prepared according to Hofmann and Hummers method. ${ }^{1}$ For the synthesis of graphene guano composite, dry guano (10 wt.\%) was mixed with graphene oxide by blade blender $(15,000 \mathrm{rpm})$ for 3 minutes. Prepared composite was reduced on doped graphene by rapid thermal treatment in nitrogen atmosphere. $100 \mathrm{mg}$ of graphene/guano composite was placed in a porous quartz capsule connected to a magnetic manipulator and inserted in a preheated furnace $\left(800^{\circ} \mathrm{C}\right)$ under nitrogen atmosphere. Thermal treatment for 10 minutes was sufficient for effective reduction of the graphene/guano composite.

Apparatus. Scanning electron microscopy (SEM) images were obtained by a JEOL 7600F field emission scanning electron microscopy (JEOL, Japan). X-ray photoelectron spectroscopy (XPS) samples were prepared by compacting a uniform layer of the materials on a carbon tape. The XPS samples were measured by a monochromatic Mg X-ray radiation source (SPECS, Germany) and a Phoibos 100 spectrometer in order to obtain survey and high-resolution C 1s spectra. All voltammetric experiments were measured using an electrochemical analyzer Autolab PGSTAT 101 (Ecochemie, Utrecht, The Netherlands) connected to a personal computer and controlled by NOVA software Version 1.8 (Metrohm Autolab B. V.).

Electrochemical measurement. Samples were dispersed in DMF to prepare a $5 \mathrm{mg} / \mathrm{mL}$ suspension for modification of the glassy carbon electrode. The GC electrode was cleaned by deionized (DI) water and polished with a $0.05 \mu \mathrm{m}$ alumina polishing cloth, and then cleaned by 
DI water again. For LSV, the prepared suspensions were sonicated for 5 min and $1 \mu \mathrm{L}$ of suspension was dropped by pipette onto the GC electrode and the dropped suspension was allowed to dry at room temperature.

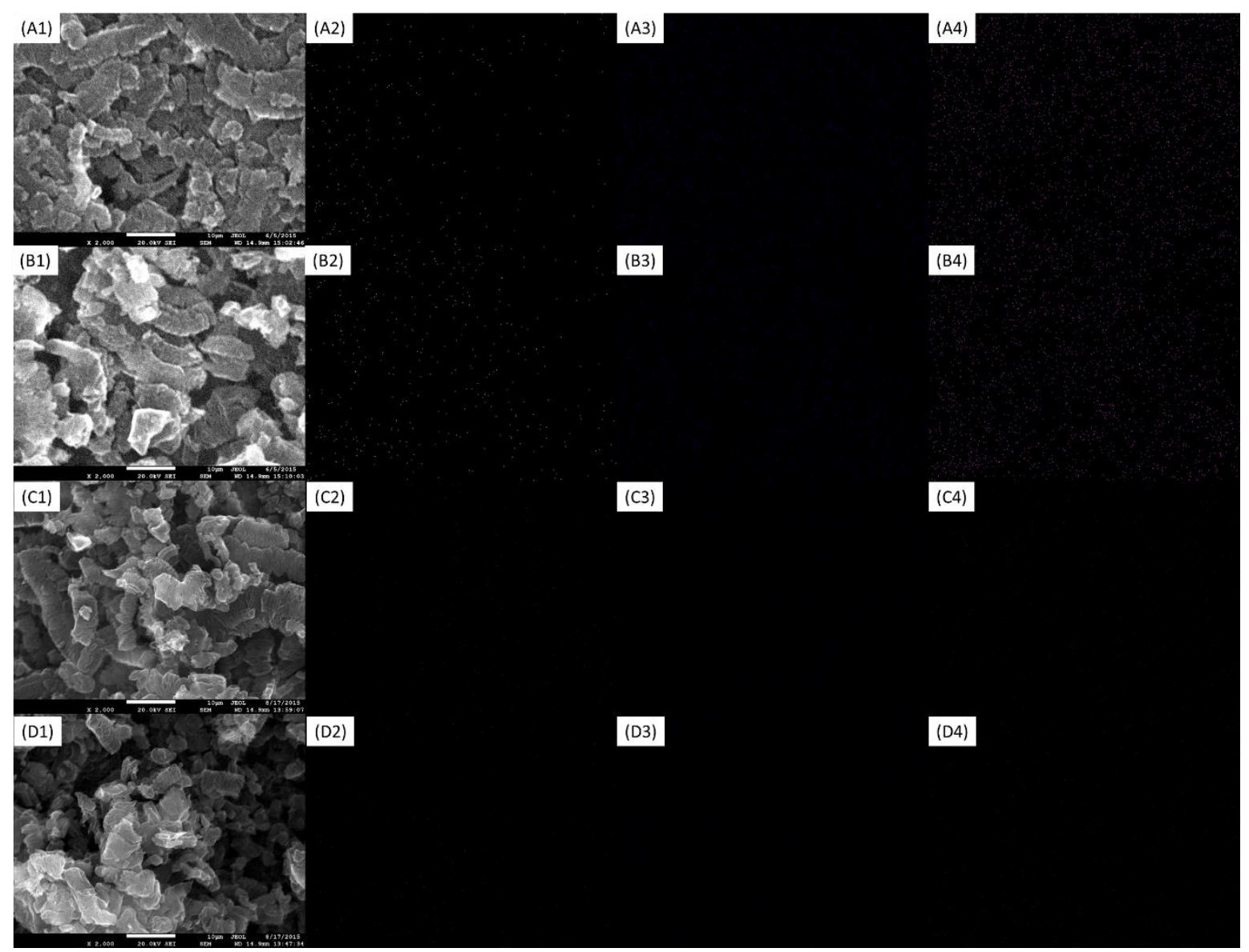

Figure S1. SEM images (column 1) and elemental mapping of $\mathrm{N}$ (column 2), $\mathrm{P}$ (column 3 ) and $\mathrm{S}$ (column 4), of (A) Ho-rGO-BD, (B) Hu-rGO-BD, (C) Ho-rGO, and (D) Hu-rGO. 


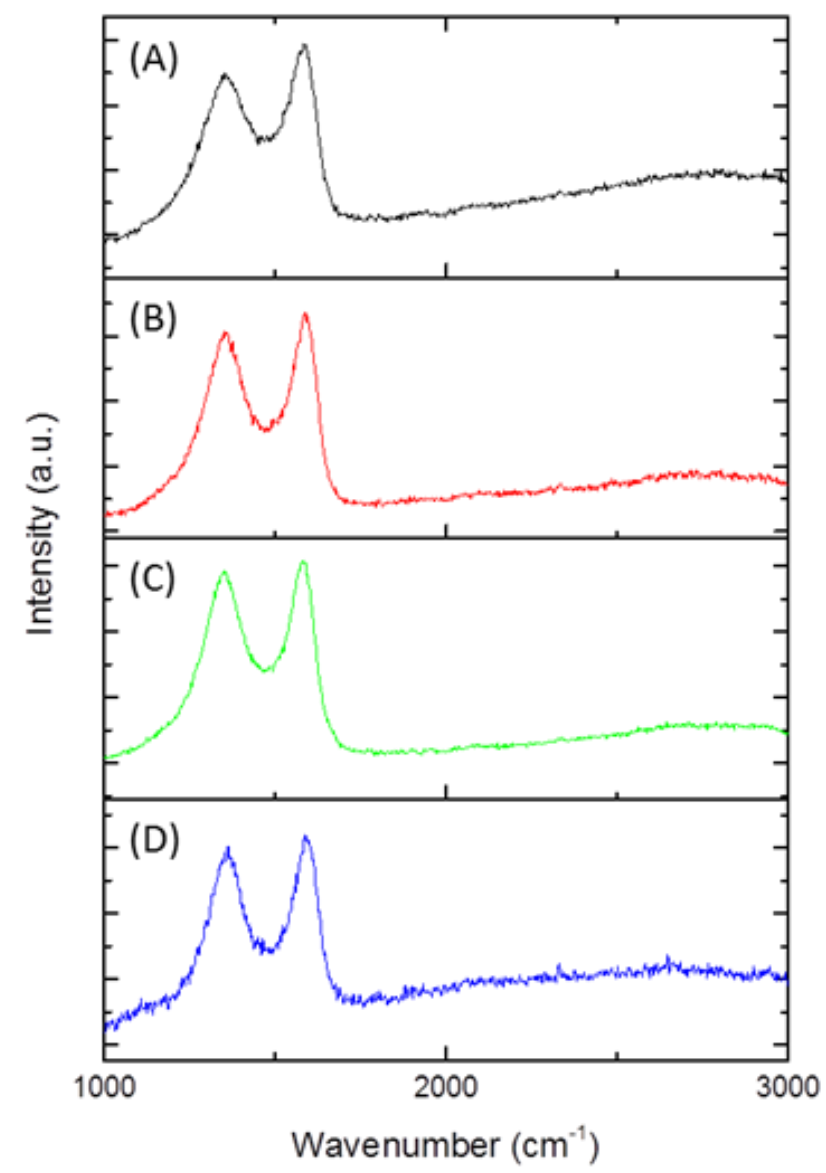

Figure S2. Raman spectra of (A) Ho-GO-BD, (B) Hu-GO-BD, (C) Ho-GO, and (D) Hu-GO. 

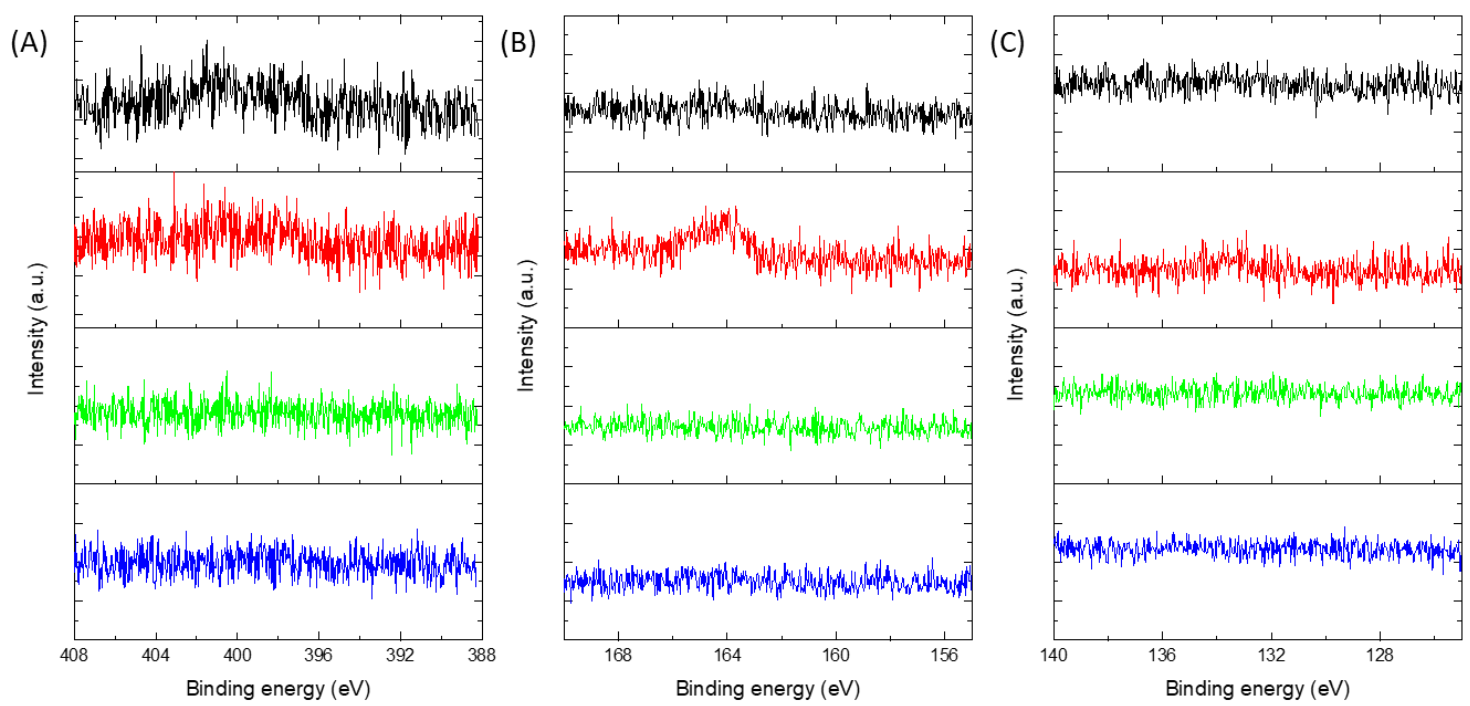

Figure S3. High resolution XPS of (A) N1s, (B) S2p and (C) P2p of Ho-rGO-BD (black), Hu-rGO-BD (red), Ho-rGO (green) and Hu-rGO (blue).

\section{Reference}

1. Hummers, W. S.; Offeman, R. E., Preparation Of Graphitic Oxide. J Am Chem Soc 1958, 80, 13391339. 